\title{
Micro-CT assessment of two different endodontic preparation systems
}

\author{
Cacio Moura-Netto(a) \\ Renato Miotto Palo(b) \\ Carlos Henrique Ribeiro \\ Camargo(b) \\ Cornelis Hans Pameijer(c) \\ Marcia Regina Ramalho da Silva \\ Bardauil(d)
}

(a) Department of Endodontics, School of
Dentistry, Univ Cruzeiro do Sul - UNICSUL,
São Paulo, SP, Brazil.

(b) Department of Endodontics, School of Dentistry, Univ Estadual Paulista - UNESP,

São José dos Campos, SP, Brazil.

(c)Department of Endodontics, School of Dentistry, Univ of Connecticut Health Center, Farmington, CT, USA.

(d) Department of Biochemistry, School of Dentistry, Univ Paulista - UNIP, São Paulo, SP, Brazil.

Declaration of Interests: The authors certify that they have no commercial or associative interest that represents a conflict of interest in connection with the manuscript.

Corresponding Author:

Cacio de Moura-Netto

E-mail: caciomn@usp.br

Submitted: Jun 01, 2012

Accepted for publication: Aug 28, 2012

Last revision: Sep 10, 2012

\begin{abstract}
The aim of this study was to compare two endodontic preparation systems using micro-CT analysis. Twenty-four one-rooted mandibular premolars were selected and randomly assigned to two groups. The samples $(\mathrm{n}=12)$ of Group 1 were prepared using the ProTaper Universal rotary system, while Group $2(\mathrm{n}=12)$ was prepared using the EndoEZE AET system complemented by manual apical preparation with K-type hand files up to \#30. A $2.5 \%$ sodium hypochlorite solution was used in both groups for irrigating. Both groups were scanned by highresolution microcomputed tomography before and after preparation (SkyScan 1172, SkyScan, Kontich, Belgium). The root canal volume and surface area was measured before and after preparation, and the differences were calculated and analyzed for statistically significant differences using ANOVA complemented by the Tukey test $(\mathrm{p}<0.05)$. The results showed no statistically significant differences between the mean volumes of dentin removal by the two systems. However, the EndoEZE AET system presented a significantly greater mean surface area compared to the ProTaper system $(\mathrm{p}<0.05)$. The EndoEZE AET system enabled preparation of a greater root canal surface area when compared to the ProTaper Universal system. There seemed to be no difference in dentin volume loss between the two systems used.
\end{abstract}

Descriptors: Dental Pulp Cavity; Root Canal Therapy; Endodontics.

\section{Introduction}

Root canal preparation is the most important phase of endodontic treatment and consists of cleaning and shaping canal space adequately. ${ }^{1}$ However, complete mechanical preparation of the root canal system is rarely achieved, because of the variations and complexity of the root canal anatomy..$^{2-4}$

In recent years, rotary instruments and oscillating systems have drawn considerable interest based on the efficacy, speed and safety of the preparation procedure.

The ProTaper Universal system (Dentisply Maillefer, Ballaigues, Switzerland) is known as having better cutting efficiency and flexibility, and especially indicated for curved canals. This NiTi alloy rotary system has six files in its pack:

- 3 shaping files for the coronal and middle third and

- 3 finishing files for the apical third. 
A study examining breakage and distortion of ProTaper, K3 Endo, and ProFile systems ${ }^{5}$ demonstrated no difference between the two groups with respect to breakage, but distortion was more pronounced for the ProFile system. The EndoEZE system (Ultradent Products, South Jordan, USA) was designed to promote a perimetric or circumferential preparation of coronal and middle thirds of root canals; however, when compared to FlexMaster, neither system was capable of completely preparing oval root canals. ${ }^{4}$ The latter system is composed of 4 stainless steel (SS) files that have a similar tip diameter $(0.10$ to $0.13 \mathrm{~mm})$ and a larger taper, starting with 0.02 of the first file to 0.06 of the last one. Because the files are made of stainless steel, it is possible to apply a brushing motion in conjunction with the $30^{\circ}$ oscillating movement of the handpiece. When using this system, preparation of the apical third must be complemented with hand files. Theoretically, oscillating systems may be more effective than rotary ones, since the latter may not reach all regions of the root canal, especially when ovalshaped canals are instrumented. This is because it is difficult to keep a rotating instrument in place, especially in the middle part of the root canal. An oscillating file, on the other hand, moves in all directions and has a short amplitude. ${ }^{4}$

Although microcomputed tomography $(\mu \mathrm{CT})$ scanning is time consuming, it has major advantag$\mathrm{es}^{6-8}$ in pre- and post-operative comparisons. $\mu \mathrm{CT}$ has a high resolution and is imminently suitable for accurately evaluating the effect of root canal preparations according to Paqué et al., ${ }^{6}$ Rhodes et al., ${ }^{9}$ and Elayouti et al. ${ }^{10}$

Assuming that preparation systems with different kinematics could lead to distinctive patterns of final preparation, these patterns may result in relatively untouched areas that probably retain bacteria and tissue remnants. The aim of this study was to compare the effect of volume change and surface area instrumentation of root canals using the rotary ProTaper Universal (PTU) system versus the oscillating EndoEZE AET (EEA) systems by means of microcomputed tomography (micro CT).

\section{Methodology Specimen selection and preparation}

This study was approved by the Research Ethics Committee of the School of Dentistry, University of São Paulo (protocol no. 225 / 2008). A total of 24 single-rooted human mandibular premolars with fully developed roots and without any previous endodontic treatment were selected for the study. The teeth were indicated for extraction for implant replacement, periodontal or orthodontic reasons. The teeth were conserved in saline solution at $4^{\circ} \mathrm{C}$. The root length of the sample ranged from 17 to $19 \mathrm{~mm}$.

\section{Acquisition and reconstruction of micro-CT images}

All the teeth were scanned by high-resolution micro-computed tomography (SkyScan 1174, SkyScan, Kontich, Belgium). The x-ray tube operated at $80 \mathrm{kV}, 10 \mathrm{~W}$ and $100 \mu \mathrm{A}$, with a $1 \mathrm{~mm}$ aluminum filter and a focal spot size of $5 \mu \mathrm{m}$. The specimen was scanned with a $90^{\circ}$-vertical axis rotation step and a single rotation step of $0.9^{\circ}$. The small sample-holder device for $\mu \mathrm{CT}$, SkyScan 1174 (SkyScan, Kontich, Belgium) was used to fit the specimen with the crown positioned downward and its long axis, perpendicular to the floor of the specimen holder and the x-ray source. The scanning time for each sample was approximately 25 min using a pixel size of $14 \mu \mathrm{m}$. The angular projections acquired with NRecon volumetric reconstruction software (SkyScan, 1.4.0, Kontich, Belgium) produced twodimensional cross-sectional slices through the root. The root canal volumes were measured in $\mathrm{mm}^{3}$ and the root canal surface areas in $\mathrm{mm}^{2}$ using the NRecon software.

\section{D and 3D analyses}

The 2D/3D analyses were performed using the CTan 1.7.0.0 version software (Skyscan, Kontich, Belgium). Select threshold levels were determined in the histogram from binary images. The white part of the binary images represented dentin (solid object), and the lower grey levels represented the root canal space for the subsequent quantitative $2 \mathrm{D}$ and 3D analyses: root canal surface areas $\left(\mathrm{mm}^{2}\right)$ and volume of canal space $\left(\mathrm{mm}^{3}\right)$ were calculated by 
the program in a tabular comma-delineated text file. Finally, CTvol 1.10.1.0 version software (Skyscan, Kontich, Belgium) was used for 3D volumetric visualization. Dentin and root canal space models were developed separately and superimposed automatically, using different colors for pre- and postinstrumented canals in order to illustrate the methodology.

\section{Experimental groups}

The teeth were randomly assigned to two groups (each group $\mathrm{n}=12$ ) according to the endodontic preparation system. The procedures described below were conducted in all samples by the same operator $(\mathrm{CMN})$, an endodontist with experience in both preparation systems. After coronal access, a size 15 K-file (Dentsply Maillefer, Ballaigues, Switzerland) was inserted into the root canal until it was visible at the apical foramen, after which the working length was established $1.0 \mathrm{~mm}$ short of the apex. A $2.5 \%$ sodium hypochlorite $(\mathrm{NaOCl})$ solution was used as an irrigating solution in both groups. In Group 1, a ProTaper Universal rotary system (Gr. PTU) was used up to an F3 file $(0.30 \mathrm{~mm}$ tip diameter and $9 \%$ larger taper). Group 2 was prepared using the EndoEZE AET system (Gr. EEA), complemented by manual apical preparation with K-type hand files up to a \#30 file. The last file of this system was the $\mathrm{S} 3$, with a $0.13 \mathrm{~mm}$ tip diameter and taper of 0.06 . The root canals were flushed with $10 \mathrm{~mL}$ of $2.5 \% \mathrm{NaOCl}$ after using each file. Afterwards, $10 \mathrm{~mL}$ of $17 \%$ ethylenediaminetetraacetic acid was used to remove the smear layer. Finally, the preparation was completed by rinsing with $20 \mathrm{ml}$ of sterile water.

All teeth were scanned again using the same protocol as described above, and the volumes and surface areas were measured after preparation. It was possible to superimpose the initial and final scan using the NRecon software, in order to illustrate the pattern of preparation for each system. All data were tabulated and the differences between initial and final volumes and areas were calculated.

The normality of the data was confirmed by the Kolmogorov-Smirnov test, and the groups were statistically compared using Analysis of Variance com- plemented by Tukey's test with a level of significance of $5 \%(\mathrm{p}=0.05)$.

\section{Results}

Table 1 shows the mean values and standard deviations of the differences between initial and final root canal volumes and surface area. ANOVA complemented by Tukey's test showed no significant differences among the mean volume changes of the two systems. However, the EEA system presented a statistically significant higher mean surface area compared to the ProTaper system $(\mathrm{p}<0.05)$.

Figure 1 illustrates the difference between the PTU and EEA systems in regard to how each system acts on the walls of root canal. The anatomy of the canal before instrumentation is indicated in

Table 1 - Mean values and standard deviations (SD) of the differences between initial and final root canal volume and surface area.

\begin{tabular}{c|c|c}
\hline Group & $\Delta$ Volume $\left(\mathrm{mm}^{3}\right)$ & $\Delta$ Area $\left(\mathrm{mm}^{2}\right)$ \\
\hline ProTaper Universal & $3.386 \pm 0.828$ & $6.052 \pm 1.596$ \\
\hline EndoEZE AET & $2.816 \pm 0.548$ & $8.509 \pm 1.951$ \\
\hline ANOVA / Tukey's test & n.s. & $\mathrm{p}<0.05$ \\
\hline
\end{tabular}

n.s.: not significant

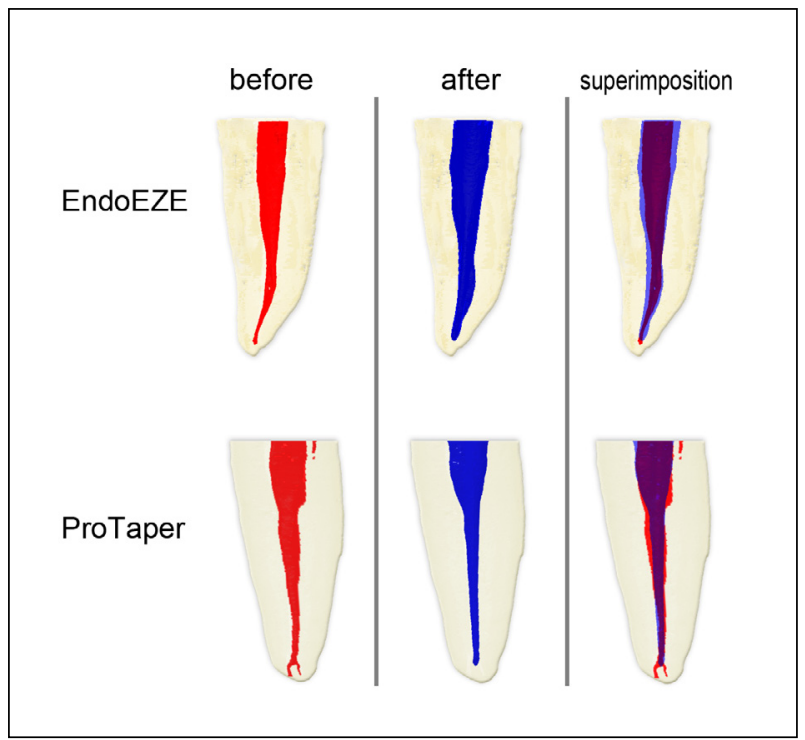

Figure 1 - Before and after instrumentation scans of the ProTaper Universal and the EndoEZE AET systems. The superimposed scans on the right demonstrate the difference in rotating and oscillation action during instrumentation. 
red, whereas blue denotes the post instrumentation configuration. On the right, a superimposition of the pre- and the post-instrumentation scans demonstrates the complete root canal instrumentation for the EEA group. In contrast, the PTU example for superimposition shows areas in red, which are not instrumented.

\section{Discussion}

Unlike the PTU system, the EEA system offers a safe method for canal instrumentation without causing instrument fractures, enabled by the mechanical action properties of its instruments. Some authors relate instrument fracture to fatigue caused by repetitive bending stresses on curved canals. ${ }^{11,12}$ Others indicate torsion as the primary mechanism responsible for fracture. ${ }^{13}$ Torsion can be generated when the rotary file is obstructed against the canal wall or exposed to excessive pressure by the clinician. ${ }^{13}$ Fractures caused by fatigue or high levels of torsion may cause failure of the endodontic treatment. ${ }^{14}$

In the present study, a comparison was made between a rotary and an oscillating system used for instrumenting root canals. The study data indicated that the EEA system prepared a larger root canal surface area in comparison to the PTU system. Stated differently, the EEA system was in contact with a larger surface area of the canal during instrumentation than the PTU system. This result means that the PTU system probably left more untouched areas than the EEA system. In our study, this difference was statistically significant. This may be attributed to a combination of alloy composition, design and mostly the movement applied by each system. The EndoEZE AET system is composed of 4 stainless steel files (SS) with almost no difference in the tip diameter $(0.10$ to $0.13 \mathrm{~mm}$ ). However, this system is composed of files of increasing taper, starting with 0.02 of the first file to 0.06 of the last one. Because the files are made of stainless steel, it is possible to apply a filing motion in conjunction with the $30^{\circ}$ oscillating movement of the handpiece. The combined action assures a more anatomical preparation, insofar as the operator can determine what part of the root canal must be more or less prepared. Because the purpose of this system is to perform the anatomical preparation, the apical enlargement must be completed with hand files up to a $0.30 \mathrm{~mm}$ diameter.

In contrast, the ProTaper Universal is a rotary system that uses NiTi alloy files that are used with an "in and out" movement combined with continuous rotation. This system prepares only the surfaces that are in contact with the file. A filing or brushing motion is not recommended, since it increases the risk of file fracture. The last file of this system (F3) has a $0.030 \mathrm{~mm}$ tip diameter and a variable taper that increases $9 \%$ with the first millimeter and then $5.5 \%$ up to the $14^{\text {th }}$ millimeter. The final preparation of this system is very similar to a taper of 0.06 . If we compare these two systems, as they were used in this study, both had a $0.30 \mathrm{~mm}$ apical diameter and a 0.06 taper at the end.

Paqué et al. ${ }^{3}$ reported similar results for EEA. However, the authors found poor results when the EEA system was used in curved canals. This is completely understandable if you imagine a 0.45 taper or a 0.6 taper stainless steel file oscillating in a curved canal. In fact, we believe that although both systems have flaws, they are highly indicated in different anatomic situations. While NiTi rotary systems are indicated for more rounded and curved anatomic situations, the EAA system is better used in flattened and isthmus areas, mostly present in the middle third of the roots. Using the ProTaper system to increase apical enlargement of curved canals did not result in complete apical preparation, but it did lead to unnecessary removal of dentin. ${ }^{10}$ Rüttermann ${ }^{4}$ reported that neither rotary nor oscillating systems produced significantly different shifts of the canal centers in the middle part of the root. Furthermore, only a few of the preparations yielded excellent results, that is, having no uninstrumented canal walls left. These authors also observed that the oscillating and rotary systems had root canals prepared only in the buccal or lingual portion of the root, as well as roots having a circular bulge with unprepared lateral extensions. ${ }^{4}$

As reported by others ${ }^{8}$ and in agreement with the findings reported here, root canal instrumentation resulted in significant gains in canal volumes 
and surface areas. The instrumentation of root canals respecting their anatomy and ensuring the least amount of dentin removal seems to be a challenging task, regardless of the system used. ${ }^{15}$

\section{Conclusions}

From the data gathered in the present study, it can be concluded that the EndoEZE system pre-

\section{References}

1. Brkanić T, Stojšin I, Zivković S, Vukoje K. Canal wall thickness after preparation with NiTi rotary files. Microsc Res Tech. 2012 Mar;75(3):253-7.

2. De-Deus G, Barino B, Zamolyi RQ, Souza E, Fonseca Jr A, Fidel S, et al. Suboptimal debridement quality produced by the single file F2 ProTaper technique in oval-shaped canals. J Endod. 2010 Nov;36(11):1897-900.

3. Paqué F, Barbakow F, Peters OA. Root canal preparation with Endo-Eze AET: changes in root canal shape assessed by microcomputed tomography. Int Endod J. 2005 Jul;38(7):456-64.

4. Rüttermann S, Virtej A, Janda R, Raab WH. Preparation of the coronal and middle third of oval root canals with a rotary or an oscillating system. Oral Surg Oral Med Oral Pathol Oral Radiol Endod. 2007 Dec;104(6):852-6.

5. Ankrum MT, Hartwell GR, Truitt JE. K3 Endo, ProTaper, and Profile systems: breakage and distortion in severely curved roots of molars. J Endod. 2004 Apr;30(4):234-7.

6. Paqué F, Ganahl D, Peters OA. Effects of root canal preparation on apical geometry assessed by micro-computed tomography. J Endod. 2009 Jul;35(7):1056-9.

7. Bergmans L, Van Cleynenbreugel J, Wevers M, Lambrechts P. A methodology for quantitative evaluation of root canal instrumentation using microcomputed tomography. Int Endod J. 2001 Jul;34(5):390-8.

8. Yang G, Yuan G, Yun X, Zhou X, Liu B, Wu H. Effects of Two nickel-titanium instrument systems, Mtwo versus ProTaper universal, on root canal geometry assessed by micro-computed tomography. J Endod. 2011 Oct;37(10):1412-6. pared a significantly larger root canal surface area in comparison to the ProTaper Universal system. However, no difference in volume of dentin removal was observed between the two systems. Based on this, it can be inferred that the EndoEZE system is capable of performing a more anatomical preparation, attaining areas that the Protaper Universal system was not capable of reaching.

9. Rhodes JS, Ford TR, Lynch JA, Liepins PJ, Curtis RV. Microcomputed tomography: a new tool for experimental endodontology. Int Endod J. 1999 May;32(3):165-70.

10. Elayouti A, Dima E, Judenhofer MS, Löst C, Pichler BJ. Increased apical enlargement contributes to excessive dentin removal in curved root canals: a stepwise microcomputed tomography study. J Endod. 2011 Nov;37(11):1580-4.

11. Grande NM, Plotino G, Pecci R, Bedini R, Malagnino VA, Somma F. Cyclic fatigue resistance and three dimensional analysis of instruments from two nickel-titanium rotary systems. Int Endod J. 2006 Oct;39(10):755-63.

12. Zinelis S, Darabara M, Takase T, Ogane K, Papadimitriou GD. The effect of thermal treatment on the resistance of nickel-titanium rotary files in cyclic fatigue. Oral Surg Oral Med Oral Pathol Oral Radiol Endod. 2007 Jun;103(6):843-7.

13. Sattapan B, Palamara JE, Messer HH. Torque during canal instrumentation using rotary nickel-titanium files. J Endod. 2000 Mar;26(3):156-60.

14. Cheung GSP. Instrument fracture: mechanisms, removal of fragments, and clinical outcomes. Endod Top. 2007 Mar;16(1):1-26.

15. Zmener O, Pameijer $\mathrm{CH}$ and Banegas G. Effectiveness of cleaning of oval-shaped root canals using Anatomic Endodontic Technology (AET), ProFile and manual instrumentation: a scanning electron microscopic study. Int Endod J. 2005 Jun;38(6):356-63. 